Figure 1. Prevalence of MOF according to BMD T-score stratified by TBS T-score

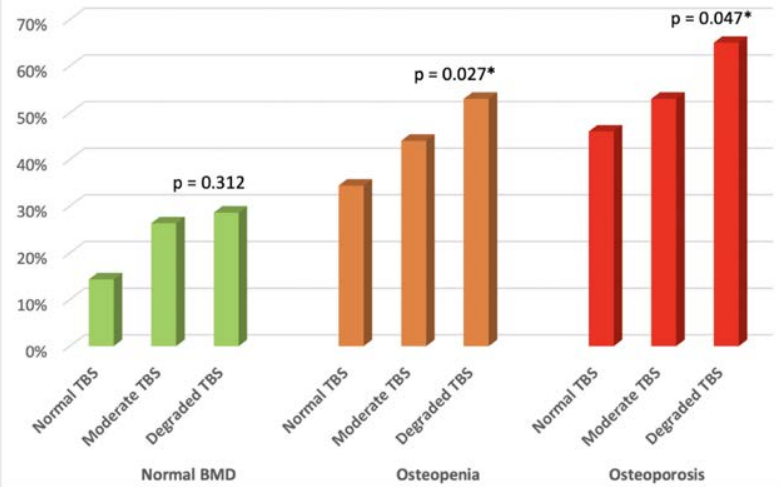

Acknowledgments: Bone density team, Robert Jones and Agnes Hunt Orthopaedic Hospital

Disclosure of Interests: None declared

DOI: 10.1136/annrheumdis-2020-eular.5585

\section{SAT0446 RISK OF ACUTE MYOCARDIAL INFARCTION (AMI) AMONG NEW USERS OF BISPHOSPHONATES: A NESTED CASE-CONTROL STUDY}

R. Mazzucchelli', S. Rodriguez-Martin ${ }^{2}$, A. García-Vadillo ${ }^{3}$, A. GarcíaLledó ${ }^{4}$, M. Gil ${ }^{5}$, F. De Abajo ${ }^{2}{ }^{1}$ Hospital Universitario Fundación Alcorcón, Rheumatology Unit, Alcorcón, Spain; ${ }^{2}$ University Alcalá de Henares, Clinical Pharmacology Unit, Alcalá de Henares, Spain; ${ }^{3}$ Hospital de La Princesa, Rheumatology Service, Madrid, Spain; ${ }^{4}$ University Hospital Príncipe de Asturias, Department of Cardiology, Alcalá de Henares, Madrid, Spain; ${ }^{5}$ Spanish Agency for Medicines and Medical Devices (AEMPS), Division of Pharmacoepidemiology and Pharmacovigilance, Madrid, Spain

Background: Evidence suggest that bisphosphonates (BF) may inhibit atherosclerosis and vascular calcification. In several small-scale clinical trials, BF improved some intermediate targets of atherosclerosis. Observational studies found a lower risk of Acute Myocardial Infarction (AMI) or stroke among BF users compared to non-users. While this epidemiological evidence suggests that BF may protect against cardiovascular events a "prevalent user bias" cannot be excluded.

Objectives: To analyze the hypothesis that BF reduce de risk of $\mathrm{AMI}$ in new users and assess whether the effect depends on the duration of treatment.

Methods: Case-control study nested in a primary cohort composed of patients aged 40 to 99 years, with at least one year of follow-up in the BIFAP database during the 2002-2015 study period. From this cohort of patients, we identified incident cases of AMI and randomly selected five controls per case, matched by exact age, gender, and index date. Adjusted odds ratios (AOR) and their corresponding $95 \%$ confidence interval $(\mathrm{Cl})$ were calculated through an unconditional logistic regression. Only new users of BF were considered.

Results: A total of 23,590 cases of IAM and 117,612 controls were included. The mean age was 66.8 (SD 13.4) years and $72.52 \%$ were male, in both groups. $584(2,47 \%)$ cases and $2,892(2.46 \%)$ controls used or had used some bisphosphonate. The use of BF was not associated with a lower risk of IAM (AOR 0.97; 95\% Cl: 0.84-1.13). Nor was it associated with the duration of treatment (AOR less than 1 year $=0.91 ; 95 \% \mathrm{Cl}: 0.72-1.15$ ); AOR more than 1 year= $1.01 ; 95 \% \mathrm{Cl}: 0.84-1.21)$. The stratified analysis by age and sex also did not show either a protective effect of BF. Detailed results by BF type are shown in the following table:

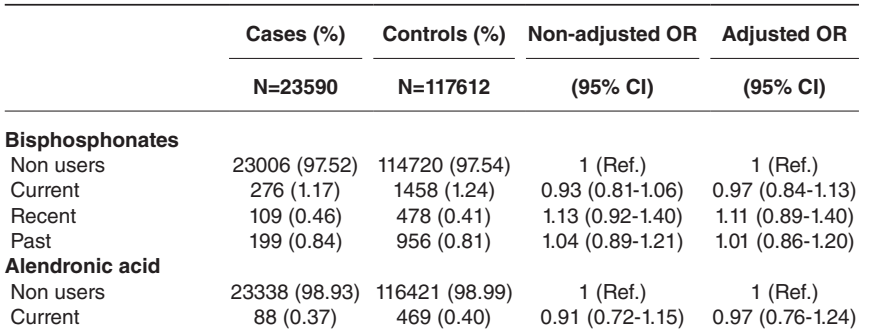

\begin{tabular}{|c|c|c|c|c|}
\hline & Cases (\%) & Controls (\%) & Non-adjusted OR & Adjusted OR \\
\hline & $\mathrm{N}=23590$ & $\mathrm{~N}=117612$ & $(95 \% \mathrm{Cl})$ & $(95 \% \mathrm{Cl})$ \\
\hline Recent & $46(0.19)$ & $201(0.17)$ & $1.17(0.84-1.61)$ & $1.18(0.83-1.66)$ \\
\hline Past & $118(0.50)$ & $521(0.44)$ & $1.12(0.91-1.37)$ & $1.13(0.91-1.40)$ \\
\hline \multicolumn{5}{|c|}{ Alendronic acid+VitD } \\
\hline Non users & 23509 (99.66) & 117155 (99.61) & 1 (Ref.) & 1 (Ref.) \\
\hline Current & $34(0.14)$ & $207(0.18)$ & $0.81(0.56-1.17)$ & $0.89(0.60-1.31)$ \\
\hline Recent & $15(0.06)$ & $77(0.07)$ & $0.94(0.54-1.63)$ & $0.71(0.40-1.29)$ \\
\hline Past & $32(0.14)$ & $173(0.15)$ & $0.92(0.63-1.35)$ & $0.83(0.56-1.25)$ \\
\hline \multicolumn{5}{|c|}{ Alendronic acid (all) } \\
\hline Non users & $23274(98.66)$ & $116057(98.68)$ & 1 (Ref.) & 1 (Ref.) \\
\hline Current & $122(0.52)$ & $671(0.57)$ & $0.89(0.73-1.08)$ & $0.95(0.77-1.18)$ \\
\hline Recent & $58(0.25)$ & $261(0.22)$ & $1.11(0.83-1.48)$ & $1.03(0.76-1.39)$ \\
\hline Past & $136(0.58)$ & $623(0.53)$ & $1.08(0.89-1.31)$ & $1.05(0.86-1.29)$ \\
\hline \multicolumn{5}{|c|}{ Ibandronic acid } \\
\hline Non users & 23504 (99.64) & 117174 (99.63) & 1 (Ref.) & 1 (Ref.) \\
\hline Current & $41(0.17)$ & $216(0.18)$ & $0.96(0.68-1.34)$ & $1.00(0.70-1.42)$ \\
\hline Recent & $18(0.08)$ & $69(0.06)$ & $1.24(0.74-2.09)$ & $1.38(0.80-2.36)$ \\
\hline Past & $27(0.11)$ & $153(0.13)$ & $0.87(0.57-1.31)$ & $0.88(0.57-1.35)$ \\
\hline \multicolumn{5}{|c|}{ Risedronic acid } \\
\hline Non users & $23355(99.00)$ & 116446 (99.01) & 1 (Ref.) & 1 (Ref.) \\
\hline Current & $102(0.43)$ & $527(0.45)$ & $0.95(0.76-1.18)$ & $0.99(0.79-1.25)$ \\
\hline Recent & $47(0.20)$ & $186(0.16)$ & $1.27(0.92-1.75)$ & $1.17(0.83-1.66)$ \\
\hline Past & $86(0.36)$ & $453(0.39)$ & $0.94(0.75-1.19)$ & $0.93(0.72-1.19)$ \\
\hline
\end{tabular}

Conclusion: Our results do not support a cardioprotective effect of BF, regardless of the duration of treatment, age, sex or background cardiovascular risk.

Disclosure of Interests: None declared

DOI: 10.1136/annrheumdis-2020-eular.741

\section{SAT0447 CORRELATES OF RADIAL BONE MICROARCHITECTURES IN OLDER ADULTS}

C. Ma ${ }^{1}$, F. Pan ${ }^{1}$, F. Wu ${ }^{1}$, H. H. Nguyen ${ }^{1}$, L. Laslett ${ }^{1}$, T. Winzenberg ${ }^{1}$, G. Jones ${ }^{1}$. ${ }^{1}$ Menzies Institute for Medical Research, University of Tasmania, Hobart, Australia

Background: Weight, dietary patterns, vitamin D, physical activity and grip strength have been suggested to be associated with bone loss in older adults. However, studies have yet been performed to investigate the associations between these factors and radial bone microarchitecture.

Objectives: This study aimed to describe the associations of weight, dietary patterns, serum 25-hydroxyvitamin D $(25(\mathrm{OH}) \mathrm{D})$ concentrations, physical activity and grip strength with bone measures in older adults.

Methods: Cross-sectional data on 201 older adults (mean age 72 years, female $46 \%$ ) from a population-based cohort study were analysed. Weight, dietary patterns, serum 25(OH)D concentrations, physical activity (steps per day), grip strength were collected and analysed from baseline to 10-year follow-up. Areal bone mineral density (aBMD) at spine, hip and whole body were measured by dual-energy X-ray absorptiometry (DXA). Radial cortical and trabecular bone microarchitectures were measured by high-resolution peripheral computed tomography (HRpQCT). Multivariable linear regression was used to analyse associations of study factors with bone measures.

Results: Weight was positively associated with radial bone area (total: $\beta=0.18,95 \% \mathrm{Cl}: 0.07,0.29$; cortical: $\beta=0.12,95 \% \mathrm{Cl}: 0.03,0.21$; trabecular: $\beta=0.18,95 \% \mathrm{Cl}: 0.05,0.32)$, and was inversely associated with compact cortical volumetric bone mineral density (vBMD) $(\beta=-0.19,95 \% \mathrm{Cl}:-0.37,-0.01)$ and trabecular thickness $(\beta=-0.25,95 \% \mathrm{Cl}:-0.43,-0.07)$. Ten-year changes in weight were not significantly associated with bone measures, apart from radial trabecular separation $(\beta=0.15,95 \% \mathrm{Cl}: 0.009,0.28)$. Western dietary pattern scores were inversely associated with radial vBMD (total: $\beta=-0.17$ 95\% Cl: $-0.32,-0.01$; cortical: $\beta=-0.19,95 \% \mathrm{Cl}:-0.34,-0.04$; compact cortical: $\beta=-0.19,95 \% \mathrm{Cl}:-0.34,-0.04$; outer transitional zone: $\beta=-0.20,95 \% \mathrm{Cl}$ : $-0.35,-0.06$ ), and were positively associated with cortical porosity (cortical: $\beta=0.18,95 \% \mathrm{Cl}: 0.03,0.33$; compact cortical: $\beta=0.19,95 \% \mathrm{Cl}: 0.04,0.34$ outer transitional zone: $\beta=0.20,95 \% \mathrm{Cl}: 0.06,0.35)$. Steps per day were not significantly associated with bone measures, apart from inner transitional zone area and thickness $(\beta=0.12,95 \% \mathrm{Cl}: 0.003,0.24 ; \beta=0.19,95 \% \mathrm{Cl}$ : $0.05,0.33)$. Healthy food pattern scores, serum $25(\mathrm{OH}) \mathrm{D}$ and grip strength were not significantly associated with radial HRpQCT measures.

Conclusion: Higher weight, but not weight change, was beneficial for radia cortical and trabecular bone area but also associated with worse compact cortical vBMD and trabecular thickness. Higher western dietary pattern scores had adverse effects on radial vBMD and cortical porosity while physical activity had inconsistent associations.

Disclosure of Interests: None declared

DOI: 10.1136/annrheumdis-2020-eular.2262 